\title{
The Physical Basis of Perceived Roughness in Virtual Sinusoidal Textures
}

\author{
Bertram Unger, Member, IEEE, Roberta Klatzky, and Ralph Hollis, Fellow, IEEE
}

\begin{abstract}
Using a high-fidelity haptic interface based on magnetic levitation, subjects explored sinusoidal textures and reported the subjective magnitude of perceived roughness. A psychophysical function was obtained spanning 33 levels of spatial periods from 0.025 to $6.00 \mathrm{~mm}$. Kinematic and dynamic variables were recorded at $1000 \mathrm{~Hz}$ and used to derive a set of variables to correlate with the psychophysical outcome. These included position, velocity, kinetic energy, instantaneous force (based on acceleration), mean force, and variability of the $z$ axis force signal from the power spectral density. The force signal was examined not only across the spectrum, but within frequency bands associated with FA1 and FA2 mechanoreceptors, and also for textures with small versus large spatial periods. The analysis implicates power of the force signal, particularly at the low frequencies associated with FA1 receptors, as the physical correlate of perceived roughness of sinusoidal textures. The relationship between power and roughness held across the range of spatial periods examined.
\end{abstract}

Index Terms-Haptics, Psychophysics, Texture, Roughness, Perception.

\section{INTRODUCTION}

$\mathbf{T}$ HE question of how humans perceive surface roughness has been of considerable interest in psychology and the neurosciences [1], [2], [3], [4], [5], [6], [7], [8] and more recently, engineering [9], [10], [11], [12], [13], [14]. Research on perception of real surfaces explored with the bare finger has described how the roughness percept changes according to surface properties such as element height, spacing, and shape (see [15] for a review). Hollins, et al. have proposed a duplex model of roughness perception, which points to the influence of different skin mechanoreceptor populations at textural scales with spatial periods below and above approximately $0.2 \mathrm{~mm}$ (microtextures and macrotextures, respectively) [16], [17], [18]. At the macro-scale, texture perception appears to rely on perception of surface geometry by SA1 mechanoreceptors, which have small receptive fields and adapt slowly. In contrast, roughness at the micro-scale appears to reflect the responses of the FA2 mechanoreceptors (also called PCs, for Pacinian Corpuscles), which have large receptive fields and adapt quickly [19], [20], [21].

Textures can be perceived not only with the bare hand but when a tool or rigid probe is used to contact the surface. In

Bertram Unger is with the Department of Medical Education, University of Manitoba, Manitoba, Canada.

E-mail: bertram.j.unger@gmail.com

Roberta Klatzky is with the Department of Psychology at Carnegie Mellon University.

Ralph Hollis is with the Robotics Institute at Carnegie Mellon University. a series of papers, Klatzky, Lederman and associates determined psychophysical functions relating perceived roughness to the spatial period of a variety of surfaces explored with a spherically tipped probe [22], [23], [4], [24]. This work provides basic data for comparison with the roughness percept of rendered surfaces that are explored with a simulated tool. In a previous paper [14] we reported psychophysical functions for roughness based on virtual textures that closely matched those findings. The critical requirement was that both the probe shape and texture in the simulation had to correspond with the physical reality, as the perceptual judgments strongly depended on probe and surface geometry. These findings served to reconcile discrepancies in the virtual-texture literature between psychophysical functions obtained with different rendering algorithms [9], [10], [12], [25].

\section{A. The Vibratory Basis of Perceived Roughness with a Probe}

When a surface at macro-textural scale is explored with the bare finger, slowly adapting receptors in the skin allow the geometry of the textural array to be sensed directly. Regardless of scale, however, use of an intervening tool means that the input to the roughness percept is vibratory in nature.

The goals of the present paper are first, to describe in detail the vibration-based signals produced when surfaces are explored with a rigid probe, and secondly, to determine which physical parameters are most related to the concomitant perception of roughness. In our experiment, subjects explored virtual sinusoidal surfaces by means of a virtual tool with a point tip, and then reported perceived roughness magnitude. A wide range of sinusoidal periods was simulated, with the result that kinematic and dynamic measures from exploration could be correlated with the roughness percept across variations in textural geometry.

A further important issue is whether the dependencies between the physical signals from vibration and roughness perception will be frequency-specific. Recall that according to the duplex model for roughness perception with the bare finger, different mechanoreceptor populations are operative at coarse and fine texture scales (SA1 and FA2, respectively). Similarly, when the textural signal comes from vibrations, as occurs if a texture is felt with a probe, two types of mechanoreceptors are implicated as possible neural mediators. In this situation, unlike the bare finger, both are fast adapting (FA), and hence vibration sensitive, but they differ considerably with respect to the frequency ranges that lead to neural excitation. The FA1 receptor has a frequency range of approximately 5-50 $\mathrm{Hz}$, while the FA2 is specialized to respond to frequencies 
above $50 \mathrm{~Hz}$ [19], [20], [21]. If perceived roughness is based on responses from a particular receptor population, only physical signals within the operative frequency band would be expected to correlate with roughness magnitude judgments. Moreover, to the extent that a particular surface induces lowor high-frequency signals, excitation of the operative receptor population(s) would be expected to vary with surface scale.

The approach of correlating perceived roughness with physical signals from exploration was adopted by Otaduy and Lin [26], [27], who rendered interactions between two textured objects. Their algorithm calculated forces and torques based on the gradient of penetration depth at a local level, under update rates on the order of $100-200 \mathrm{~Hz}$ with 5-10 contact patches between objects. Using a model of human dynamics as an input to their model, they were able to demonstrate, in simulation, that maximum acceleration of the probe followed a quadratic function over element spacings. The function varied with probe diameter, applied force and exploratory speed in ways that were qualitatively similar to the psychophysical studies of the same factors by Klatzky et al. [23]. In another related study, Yoshioka et al. [28] elicited roughness, hardness and stickiness ratings, along with similarity comparisons, with both direct and indirect touch for 16 natural textures. Vibratory measurements were also obtained for each surface under conditions of passive scanning at a fixed rate of $40 \mathrm{~mm} / \mathrm{s}$. They determined that vibratory power correlated well with perceived roughness. Previous studies had implicated the PC mechanoreceptors in the sensation of fine texture under direct touch and had demonstrated a correlation between vibratory power, filtered by a function describing the PC frequency sensitivity, with roughness [29], [30]. Yoshioka et al. found that raw vibratory power correlated slightly better than filtered for indirect touch, although the results were almost indistinguishable. However, the study is limited by the freely varying nature of the stimuli and by the fact that vibrations were measured under restricted contact conditions, separately from the exploration that resulted in judged roughness.

The present study was able to more systematically address the issue of the physical underpinnings of perceived roughness from vibration, by using a high-fidelity haptic display based on magnetic levitation technology. The device allowed surfaces to be rendered with high stiffness across a range of sinusoidal periods spanning 0.025 to $6.00 \mathrm{~mm}$. The kinematics and dynamics of exploration were recorded at a rate of $1000 \mathrm{~Hz}$ during natural exploration. The results differentiate among a number of candidate physical variables that potentially underlie the roughness percept and further examine the frequency specificity of the observed relationship. To preface our results, the study confirms the importance of power in the force signal perpendicular to the surface, and somewhat surprisingly, implicates FA1 mechanoreceptors across the range of simulated surfaces.

\section{EXPERIMENTAL SETUP}

\section{A. Magnetic Levitation Haptic Device}

Our experiment employed a 6-DOF magnetic levitation haptic device (MLHD) using Lorentz forces [31], [32], [33] that is capable of rendering virtual textures with high fidelity. As shown in Fig. 1, the device features a manipulandum that is rigidly attached to a lightweight hemispherical flotor. The flotor has six spherical coils that interact with strong magnetic fields that enable it to levitate without friction and without contact with its surroundings. The six Lorentz forces generated by the coils combine to exert a 6-wrench on the manipulandum. The position and orientation of the flotor is tracked by optical sensors. A closed-loop servo algorithm allows stiffness and viscosity in all axes to be controlled over a wide range of values. The device has a $-3 \mathrm{~dB}$ bandwidth of approximately $120 \mathrm{~Hz}$ with smooth roll-off to nearly a $\mathrm{KHz}$. Advanced versions of the device have been commercially available since 2008 .

For our experiments, a proportional-derivative (PD) controller running on an AMD 2100+ processor controlled the device with a servo update rate of $1000 \mathrm{~Hz}$, proportional gains set to $10 \mathrm{~N} / \mathrm{mm}$ in translation and $25 \mathrm{Nm} /$ radian for orientation, and derivative gains set to $0.04 \mathrm{~N} / \mathrm{mm} / \mathrm{s}$ in translation and $0.5 \mathrm{~N} / \mathrm{radian} / \mathrm{s}$ for rotation. These gain settings provided a relatively stiff surface and prevented, to a large extent, rotation of the manipulandum, which was desirable as only $z$-axis forces were actively generated by the rendering algorithm. The force of gravity on the manipulandum was reduced by an opposing feed-forward force of $5 \mathrm{~N}$ that reduced the weight of the flotor from approximately 580 grams to 70 grams. More details about the device are available in [14].

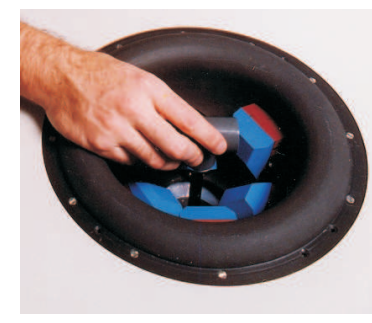

(a)

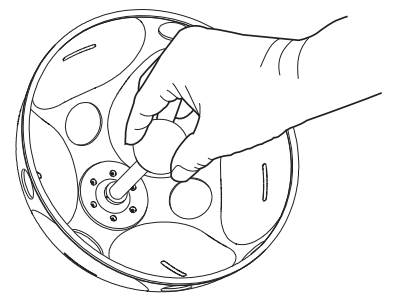

(b)
Fig. 1. Magnetic Levitation Haptic Device (MLHD) used in the experiments: $(a)$ photo showing hand and manipulandum, $(b)$ diagram showing levitated hemispherical flotor with embedded coils interacting in strong magnetic fields.

\section{B. Texture Simulation}

The experimental stimuli were sinusoidal grating textures (SGTs). The rendering algorithm treated the haptic interaction point (HIP) as an infinitely small probe that was mapped onto a surface, the height of which ( $z$-axis) varied as a sinusoidal function of distance along the $x$ axis. Width ( $y$ axis) was constant. The orientation of the manipulandum was controlled to keep it vertical at all times. Contact of the probe with the surface generated a $z$-axis force proportional to, and directionally opposed to, its penetration depth. When the probe was not in contact with the surface, no forces were actively generated, so that the probe was subject only to the reduced gravitational force.

Thirty-three virtual SGTs with spatial periods ranging from 0.025 to $6.00 \mathrm{~mm}$ were generated according to the algorithm 
described above. The sinusoid amplitude was $0.4 \mathrm{~mm}$ peakto-peak, consistent with the height of texture elements investigated by other studies [23]. The smallest grating periods approached the resolution of the MLHD (5-10 microns). The largest grating periods allowed 4 spatial periods within the MLHD's workspace. The period space was sampled asymmetrically, with a larger number of samples from the shorter periods, as can be seen from the $x$ axis results shown in Fig. 2.

An important issue is whether the MHLD is capable of rendering textures with very small periods. Modeling of the device using measurements of its damping and spring coefficients shows that the frequency response has a $\pm 3 \mathrm{~dB}$ corner at approximately $120 \mathrm{~Hz}$ with slow roll off, at typical gain settings. This will lead to attenuation of the MLHD's positionfollowing capabilities when the device is required to rapidly traverse sinusoidal gratings with small periods $(\leq 0.2 \mathrm{~mm})$. Another issue is whether, given subjects' typical movement speed (reported below as on the order of $25-30 \mathrm{~mm} / \mathrm{s}$ ), the MLHD is capable of producing the range of frequencies required to simulate the sinusoidal period. Since the rate for servoing the device and sampling data is $1000 \mathrm{~Hz}$, the Nyquist Rate implies that, for the smallest periods encountered, the expected frequencies $(>500 \mathrm{~Hz})$ are greater than those the device can accurately reproduce. For periods greater than $0.2 \mathrm{~mm}$, the device should be capable of following a sine wave without significant attenuation. Although finer textures approach the limitations of the device, roughness-estimation data reported below show no evidence of an attenuation in the region below spatial periods of $0.2 \mathrm{~mm}$.

\section{Experimental Design and Procedure}

The participants were 27 students associated with the psychology department at Carnegie Mellon University, who received credit for participation, or paid and unpaid student volunteers from other units at Carnegie Mellon. Procedures for informed consent were used in compliance with University review, and the project was approved by Carnegie Mellon's ethics board. All subjects used the right hand for exploration with the MLHD.

Subjects were seated approximately $500 \mathrm{~mm}$ from a graphical display used for responses but not texture displays. They listened to white noise via headphones to prevent auditory cues to texture. They freely wielded the MLHD manipulandum, except for a warning that excessive force would cause the device to shut down. After exploration, they gave an estimate of the roughness magnitude of the explored surface by entering a non-zero number that reflected its roughness on a computer keypad. They were instructed that larger values should correspond to greater roughness magnitude, but no scale was imposed and no standard was given. The MLHD manipulandum position and force data were recorded throughout the experiment at $1000 \mathrm{~Hz}$.

The sequence of experimental trials consisted of 33 textures, presented 4 times each in random order, for a total of 132 recorded trials per subject. A preliminary demonstration block was included, representing the range of texture to be experienced in random order.

\section{PSychophysical FunCtion OF ROUGHNESS MAGNITUDE}

The psychophysical function relating perceived magnitude to experimentally manipulated variables were calculated for each subject. Outliers with values greater than ten times a subject's overall median response were removed before further analysis. Because the subject chose his or her own magnitude estimation scale with which to represent roughness, it was necessary to normalize the reported values before generating this function. For this purpose each observation was divided by the mean of all observations for that subject, then re-scaled by multiplying it by the mean over all subjects. The 4 values for each spatial period were then averaged for each subject and used for statistical evaluation.

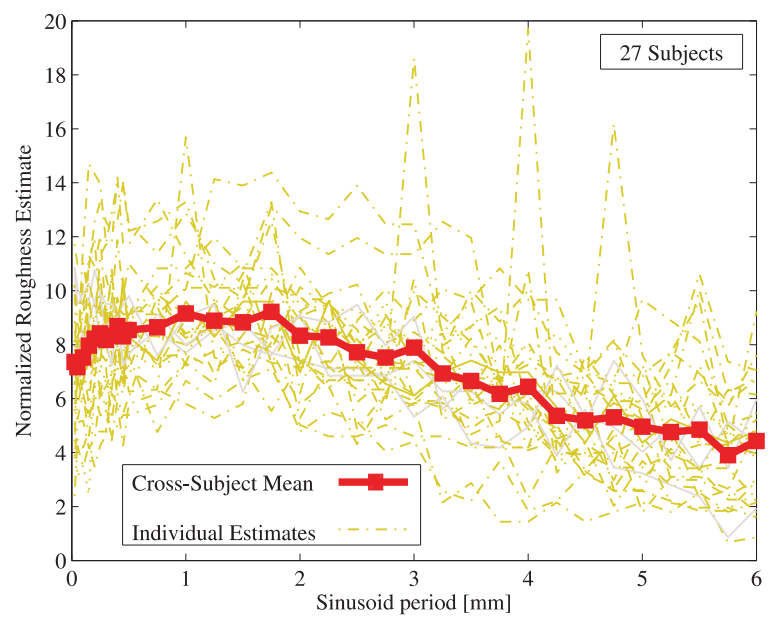

Fig. 2. Plot of individual normalized roughness psychophysical functions for 27 subjects superimposed on their cross subject mean. Reprinted from [14], copyright IEEE Computer Society

Superimposed plots of the psychophysical roughness function for each subject as well as the mean function can be seen in Fig. 2. Although the functions show considerable variance between individual subjects, most follow a pattern of an initial rise followed by a long decline in roughness as a function of increasing texture period. A one-way ANOVA found that element spacing had a significant effect on reported magnitudes $(\mathrm{F}(32,726)=11.52, \mathrm{p}<0.0001)$. Note that any limitations in rendering textures with very small periods are not apparent in the data, as roughness for sinusoid periods less than $0.2 \mathrm{~mm}$ is not particularly low.

In [14] we characterized the function as bi-partite and attributed its behavior to either or both of two potential causes: a transition in the physical property leading to perceived roughness at this spacing, and/or a transition in the underlying neural processing. However, a fuller understanding of the perceived roughness of sinusoidal surfaces requires a detailed analysis of the signals generated by the probe/texture interaction, which is the main focus of this paper. We begin with an analysis of the physical parameters of the stimulus that may be responsible for the perception of roughness in probebased texture exploration. We then consider the implications of the data for the receptor population that might mediate roughness perception of rendered sinusoidal surfaces. We 
focus in particular on mechanoreceptors that are thought to respond to relatively slow vs. fast vibrations (FA1 and FA2).

\section{Physical Parameters of Exploration And RELATION TO ROUGHNESS}

To affect roughness, geometric properties of the surface, together with exploratory motions, must lead to variations in the physical inputs to the receptors in the hand. In this section the kinematic data from the MLHD, captured during magnitude estimation trials, will first be examined to determine how probe position, velocity and acceleration change with sinusoidal period, and to assess whether one or more of these variables might account for the resulting variations in roughness estimates. We then consider the degree to which roughness correlates with dynamic physical properties, including force variability, mean force, kinetic energy and power in the force signal. (Note that due to loss of MLHD data for 4 subjects through computer error, this data analysis incorporates 23 of the 27 subjects for whom roughness magnitude estimations were reported.)

\section{A. Texture Exploration: Position}

We initially describe probe position data, although position per se (i.e., as cued by sustained skin deflection or signals to muscles, tendons and joints) is not a likely candidate for the percept of roughness, given that the receptors related to texture respond best to changing stimuli [18].

Figure 3 shows typical data for the position of the haptic device manipulandum during a single trial on two different surfaces. The large sinusoidal motions are due to subjects' hand motion back and forth along the $x$ axis as they explore the sinusoidal grating. Although the ridges and grooves of the texture extend along the $y$ axis, there are smaller sinusoidal motions along this axis with the same frequency as the $x$ axis motion. These result from the fact that motion of the manipulandum has an angular deviation relative to the $x$ axis; the difference in phase between $x$ - and $y$-axis motion is due to a slight arc of the manipulandum during the sweep.

Of greatest interest is the motion on the $z$ axis, which constitutes the rise and fall of the probe as determined by the interaction between the subject's hand, the device, and the texture presented. In this experiment a sinusoidal pattern with an amplitude determined by the penetration depth algorithm might be expected if the HIP precisely followed the textured surface. Examining Fig. 3 it can be seen that this is clearly not the case, especially for sinusoids with small periods.

The deviations in the $z$-axis path from a pure sinusoid might reflect the fact that the HIP was not constrained to stay on the texture surface. Thus subjects might elect to lift it above the texture or it could fly above the surface due to dynamic effects. As well, the position of the HIP is determined by the penetration depth algorithm subject to the force applied by the subject, which might vary with time and hand position. Third, particularly for the textures with a spatial period below 0.2 $\mathrm{mm}$, device resolution and frequency response could prevent accurate haptic display of the required position, as described above.

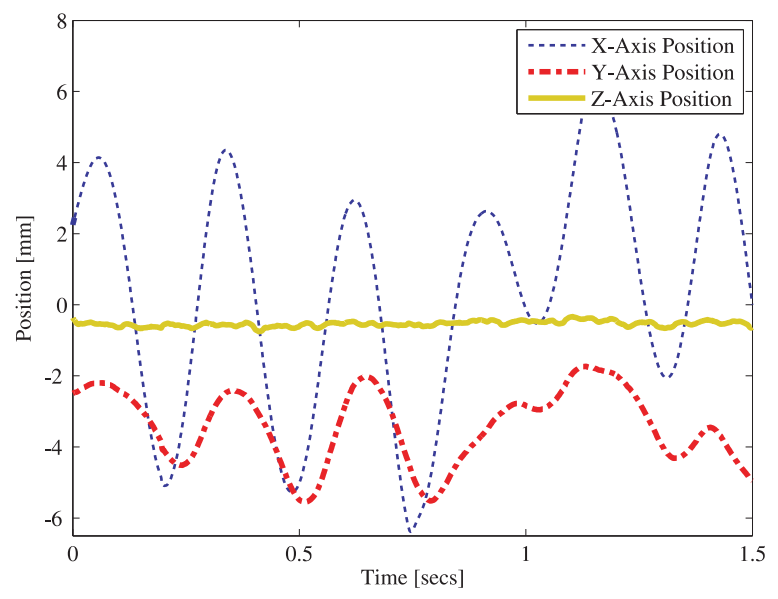

(a)

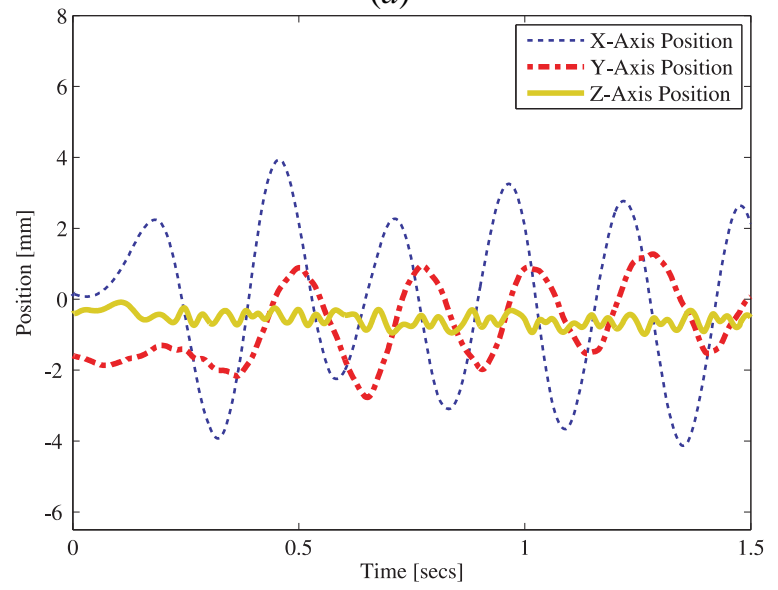

(b)

Fig. 3. Representative example of manipulandum motion along $x, y$ and $z$ axes during a single subject trial on sinusoidal grating texture with a period of $(a) 0.025 \mathrm{~mm},(b) 2.5 \mathrm{~mm}$.

\section{B. Texture Exploration: Velocity}

We next turn to the velocity of the probe as it moves across the surface, which determines the temporal frequency with which texture elements are encountered and hence the change in position of the probe against the skin with respect to time. The mean absolute instantaneous velocities, determined from the first derivative of position recordings of the HIP along each axis, are shown as a function of sinusoid period in Fig. 4. Angular velocities about each axis are shown in Fig. 5, A series of 1-way ANOVAs revealed no effect of sinusoid period on the velocity along or about any axis except $z$ (see Table I).

\begin{tabular}{|c|c|c|}
\hline Velocity & $\mathrm{F}(32,726)$ & $\mathrm{p}$ \\
\hline$x$-axis & 0.27 & $>0.05$ \\
$y$-axis & 0.22 & $>0.05$ \\
$z$-axis & 7.16 & $<0.001$ \\
Roll & 0.26 & $>0.05$ \\
Pitch & 0.30 & $>0.05$ \\
Yaw & 0.23 & $>0.05$ \\
\hline
\end{tabular}

TABLE I

1-WAY ANOVA RESULTS FOR EFFECT OF SINUSOID PERIOD ON MANIPULANDUM LINEAR AND ANGULAR VELOCITY. ONLY $z$-AXIS VELOCITY SHOWS A SIGNIFICANT EFFECT. 


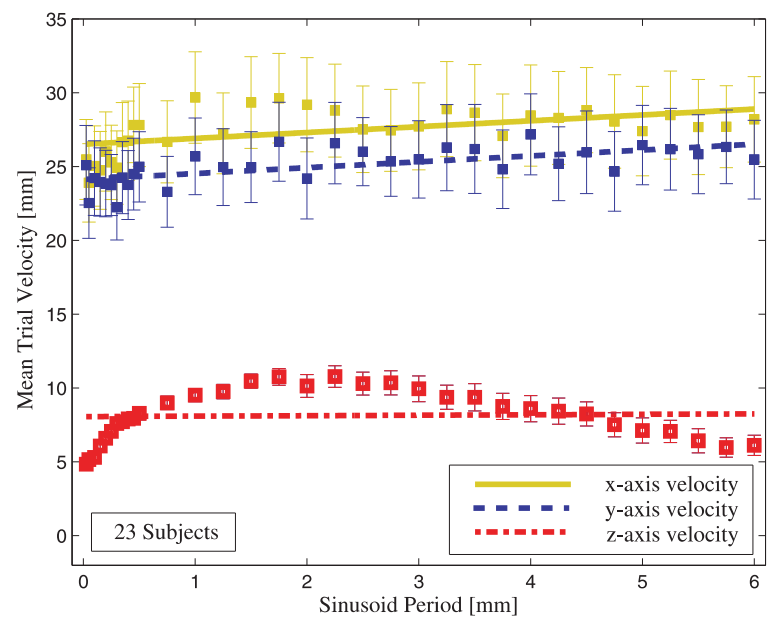

Fig. 4. Cross subject mean trial velocity as a function of sinusoidal grating period with linear fits for $x, y$, and $z$ axes.

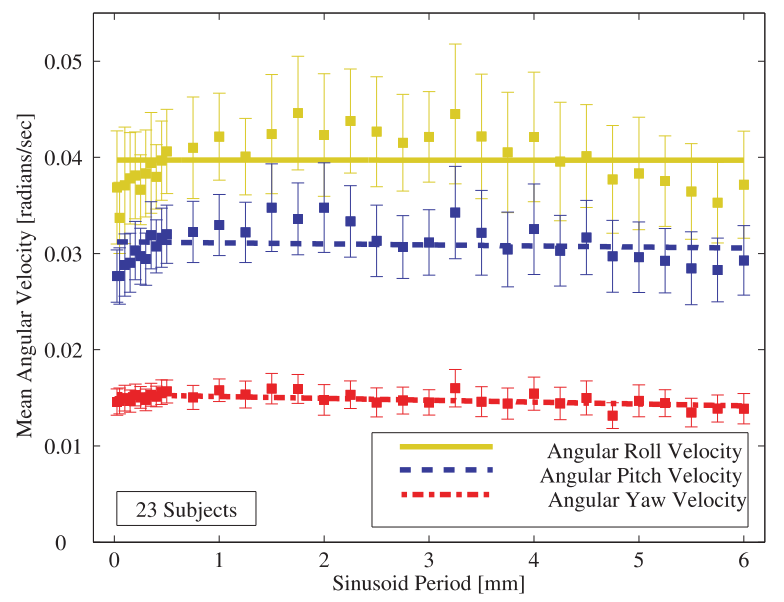

Fig. 5. Mean angular velocity for roll, pitch and yaw in radians/s. A linear fit is plotted to each set of data.

It is not surprising that angular velocity was essentially negligible and did not vary with period, since the device was constrained in rotation. In contrast, the $x$-axis and $y$ axis motion was freely controlled by the subject. Of particular interest is the finding that during unconstrained motion, the $x$-axis velocity was essentially invariant, despite the fact that the height of the rendered sinusoid varies along this axis. Apparently, the subject's movement speed was not affected by the shape of the sinusoid. The fact that the roughness magnitudes were clearly not constant over spatial period, while velocity was approximately constant, is a strong indication that velocity is not the controlling factor in perceived roughness.

For a given stimulus explored at a constant rate, the temporal frequency with which sinusoidal peaks are encountered as a probe moves is simply the $x$ velocity divided by the sinusoidal period for that stimulus. An implication of the constancy of the $x$-axis velocity observed here is that the subject experiences something close to this ideal frequency, at least on average. (In practice, the frequency would depend locally on movement speed and probe trajectory.) Log average temporal frequency would then be related to log spatial period with a slope of
-1 , which was true of the present data (see Fig. 6). The fact that roughness magnitudes did not follow this linear log-log trajectory means that temporal frequency can be excluded, along with $x$ velocity, as the factor that governs perceived roughness.

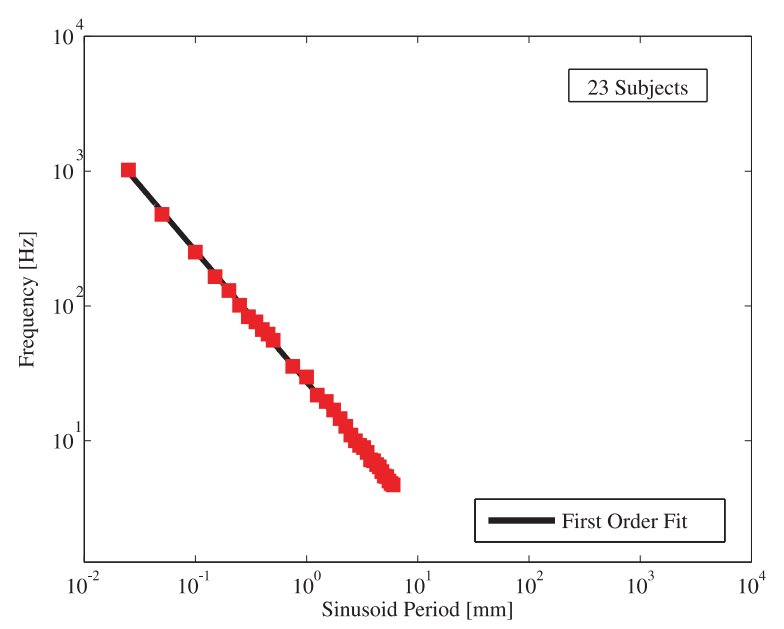

Fig. 6. Log-log plot of temporal frequency (mean over subjects) encountered by subjects versus sinusoidal grating period with first order fit. Slope $=-0.977 \pm 0.008$, Y-intercept $=1.372 \pm 0.051, R^{2}=$ 0.99 . Temporal frequency is calculated as a subject's mean $x$-axis velocity divided by the sinusoidal grating period.

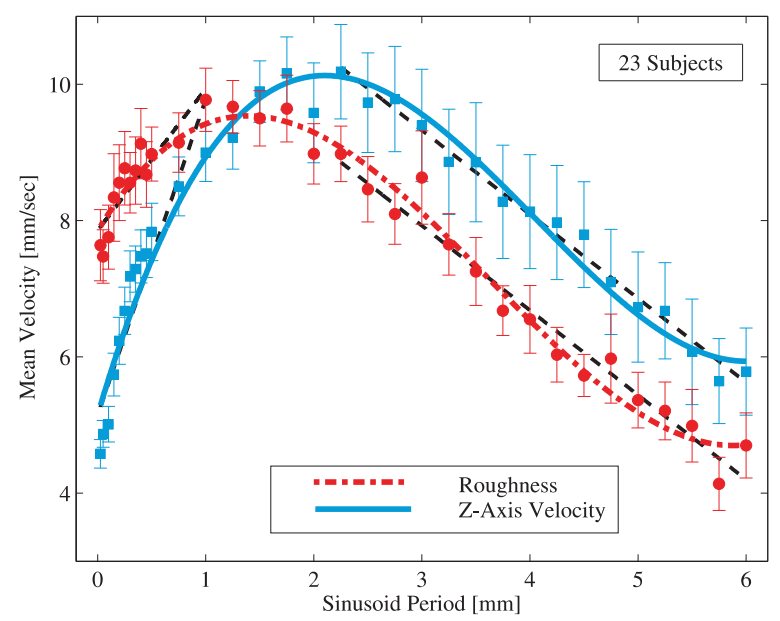

Fig. 7. Cross subject mean trial velocity and normalized subject roughness estimates as a function of sinusoidal grating periods. A third order fit to each complete data set is shown. $\left(R^{2}=0.97\right.$ and 0.95 for roughness and velocity respectively). A linear fit to the data is also shown for small periods of $0.025-1.00 \mathrm{~mm}$ and for large periods of 2.25-6.0 mm. (Ascending Roughness Fit $R^{2}=0.80$, Ascending Velocity Fit $R^{2}=0.87$, Descending Roughness Fit $R^{2}=0.9599$, Descending Velocity Fit $R^{2}=0.99$.)

Only velocity along the $z$-axis showed a significant relation to spatial period (see Fig. 4), $\mathrm{F}(32,726)=7.16, p<.001$. This makes the $z$ velocity a candidate for the physical factor that governs perceived roughness. The velocity function increased rapidly with increasing period, then decreased more slowly over the rest of the range of spatial period. This mimics the pattern of the roughness function, although the latter peaks slightly earlier along the spatial-period axis. 
Quantitative comparison of the effect of spatial period on $z$-axis velocity to that on subjective roughness estimates is shown in Fig. 7. Note that in this and the following figures where roughness is compared to a physical predictor, for ease of comparison the roughness function has been re-scaled so that its mean matches that of the predictor. (The original normalization of roughness means that its source scale is irrelevant.) Clear similarities can be seen in the shape of the two fit curves. The slope of a straight line fit to the linear ascending portion of the roughness data was $2.08 \mathrm{~mm} / \mathrm{s}$ per millimeter of period $\left(\mathrm{mm} / \mathrm{s} / \mathrm{mm}_{\text {period }}\right)$. A similarly fit line for the velocity curve had a slope of $4.64 \mathrm{~mm} / \mathrm{s} / \mathrm{mm}_{\text {period }}$. The linear fits to the descending portions of the two curves showed even greater similarity, with a descending slope of -1.23 and $1.24 \mathrm{~mm} / \mathrm{s} / \mathrm{mm}_{\text {period }}$ for roughness and velocity, respectively. However, the fit curves different substantially in the texture period where they peak (roughness at $1.39 \mathrm{~mm}$, velocity at $2.10 \mathrm{~mm}$ ). We will return to consideration of velocity as a predictor, in comparison with other variables.

\section{Texture Exploration: Kinetic Energy}

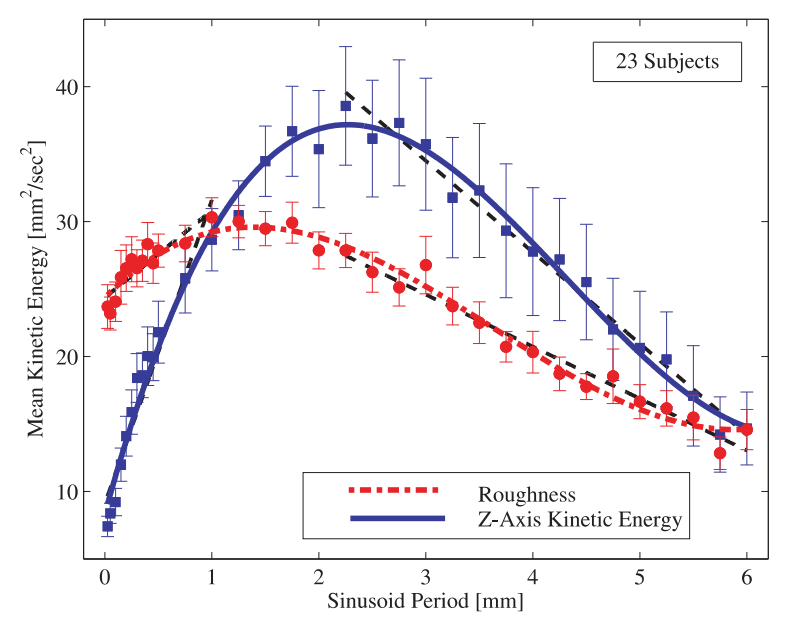

Fig. 8. Mean $z$-axis kinetic energy compared to roughness as a function of sinusoid period. A third order fit to each data set is shown with $R^{2}=0.99$ for the kinetic energy and $R^{2}=0.97$ for roughness. A linear fit to the data is also shown for small periods of $0.025-1.00$ $\mathrm{mm}$ and for large periods of 2.25-6.0 mm. (Ascending Roughness Fit $R^{2}=0.80$, Ascending Velocity Fit $R^{2}=0.93$, Descending Roughness Fit $R^{2}=0.96$, Descending Velocity Fit $R^{2}=0.98$.)

While $z$-axis velocity is problematic as a basis for roughness judgments, the kinetic energy, which is proportional to the square of velocity, is also a potential candidate. The kinetic energy, $K E$, for a mass, $m$, moving with velocity, $v$, is typically calculated as

$$
K E=\frac{m v^{2}}{2} .
$$

The moving mass, in this case, comprises the mass of the flotor. As this is a constant (581 grams) in our case, the relationship between kinetic energy and the geometry of the sinusoidal period depends entirely on $v^{2}$ alone. In Fig. 8 a plot of kinetic energy as a function of sinusoid period can be seen, along with a plot of subjects' roughness estimates at the same periods (rescaled as described above). A 1-way ANOVA showed a significant effect of sinusoid period on mean kinetic energy $(F(32,726)=7.62, p<0.001)$. The slopes of straight lines fit to the ascending portion of the roughness and kinetic energy curves were 6.45 and $22.5 \mathrm{~mm}^{2} / \mathrm{s}^{2} / \mathrm{mm}_{\text {period }}$, respectively. Lines fit to the descending portion of the curves had slopes of -3.85 and $-6.78 \mathrm{~mm}^{2} / \mathrm{s}^{2} / \mathrm{mm}_{\text {period }}$ respectively.

While the velocity function appeared, on inspection, to be close to the shape of the roughness function, albeit shifted in phase, the kinetic energy function in Fig. 8 differs substantially from that of roughness. The slope of its linear portion is dissimilar, and it is no closer in phase to the psychophysical function for roughness than the velocity function. Kinetic energy is therefore unsuited as the underlying physical factor which results in a perception of roughness.

\section{Texture Exploration: Force}

Another possible physical property that might account for roughness perception is the force the haptic device exerts on the subject's fingers. One way to analyze force is to examine the effects of acceleration, since force is related to the acceleration by $F=m a$, where $\mathrm{m}$ is the mass of the flotor and manipulandum. As this is constant, if one assumes that the user exerts a relatively constant force (consisting of the weight of their arm and hand plus applied muscular force), acceleration can be used as a surrogate for the resultant forces experienced by the subject. (This assumption is supported by a finding reported below that mean $z$-axis force is essentially constant over spatial period.) Here we compute acceleration from the second derivative of the instantaneous MLHD manipulandum position, recorded at $1 \mathrm{KHz}$.

A plot of mean $z$-axis acceleration, along with roughness estimates, is shown in Fig. 9. A clear relationship between roughness and mean instantaneous acceleration can be seen, although acceleration peaks at a much smaller texture period $(0.3 \mathrm{~mm}$ from a third-order fit) than that of roughness (1.39 $\mathrm{mm})$. The slopes of the nearly linear ascending portions of the function differ by nearly a factor of two (730.8 and 470.3 $\mathrm{mm} / \mathrm{s}^{2} / \mathrm{mm}_{\text {period }}$ for acceleration and roughness respectively) but are nearly the same for the linear fits to the descending portions $\left(-89.1\right.$ and $-118.6 \mathrm{~mm} / \mathrm{s}^{2} / \mathrm{mm}_{\text {period }}$ for acceleration and roughness respectively). Given the phase difference, as with velocity, caution is indicated in inferring that instantaneous force accounts for roughness judgments.

As an alternative to inferring force from acceleration, it is also possible to look directly at the forces commanded by the MLHD in response to the depth of penetration of the HIP below the texture. Mean force averaged approximately $10 \mathrm{~N}$, including the feed-forward force of $5 \mathrm{~N}$, across subjects. However, unlike the instantaneous force as inferred from acceleration, the mean $z$-axis force was virtually invariant across sinusoidal period; by 1 -way ANOVA $(\mathrm{F}(32,726)=0.05$, $\mathrm{p}>0.05$. Note that wheras mean force pools the force applied during a trial, mean acceleration yields a measure of the average instantaneous force, or force variability experienced by subjects during that time. This suggests that force variability may be critical to perceived roughness, as is explored next. 


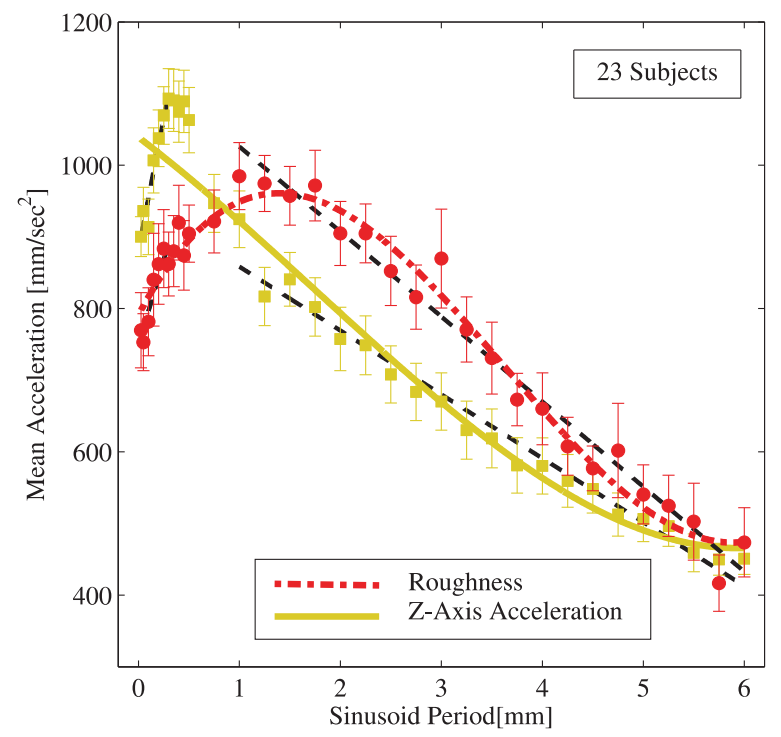

Fig. 9. Mean $z$-axis acceleration compared to roughness as a function of sinusoid period. Acceleration and roughness are normalized for comparison. A third order fit to each data set is shown with $R^{2}=$ 0.94 for acceleration and $R^{2}=0.97$ for roughness. A linear fit to the data is also shown for small periods of $0.025-0.3 \mathrm{~mm}$ and for large periods of 1.0-6.0 mm. (Ascending Roughness Fit $R^{2}=0.85$, Ascending Acceleration Fit $R^{2}=0.93$, Descending Roughness Fit $R^{2}=0.97$, Descending Acceleration Fit $R^{2}=0.97$.)

\section{E. Texture Exploration: Power}

We next consider the force signal's power (its variability) as a candidate for the variable mediating roughness perception. Taking the power spectral density (PSD) of the force signal using a periodogram technique with 1024 Fast Fourier Transform points, a PSD periodogram for frequencies from $1-500 \mathrm{~Hz}$ was generated. Since the maglev commanded force is sampled at $1000 \mathrm{~Hz}$, the Nyquist frequency limits the useful signal to $500 \mathrm{~Hz}$. Preliminary analysis of the periodograms showed that regardless of texture period, most of the power in the signal was found below $100 \mathrm{~Hz}$, being particularly concentrated in the band from 5-30 Hz. Total power peaked around a period of $2-3 \mathrm{~mm}$. The sensitivity to spatial period, discussed further below, suggests that the total power in the force signal over the range measured (i.e., below $500 \mathrm{~Hz}$ ) may be the salient physical factor perceived as roughness.

A plot of the total power in the PSD periodogram (i.e., in the $z$-axis force signal) over the range of sinusoidal texture periods, averaged over subjects, can be seen in Fig. 10, together with the psychophysical roughness function (rescaled). A 1way ANOVA showed significant effects of sinusoid period on power $(\mathrm{F}(32,726)=7.58, \mathrm{p}<0.001)$. The maximum roughness and maximum force-signal power occurred at the same texture period $(1.39 \mathrm{~mm})$ while the slopes of linear fits to the ascending and descending portions of the functions were very similar, particularly, in the descending limb (see figure caption for values).

\section{F. Power vs. Other Physical Parameters}

To compare the various parameters, correlation coefficients were computed between the psychophysical function for

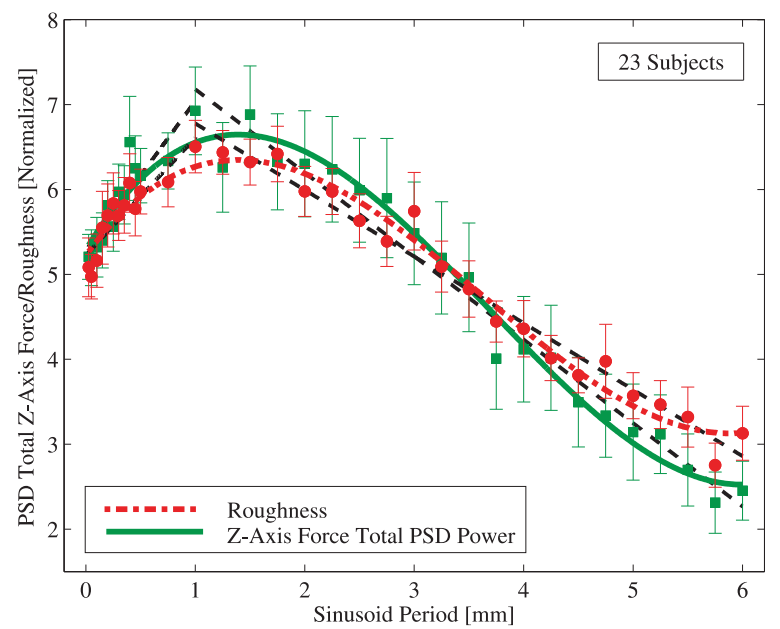

Fig. 10. Total power of $z$-axis force signal from the power spectral density compared to roughness as a function of sinusoid period. Power and roughness are normalized for comparison. A third order fit to each data set is shown with a maximum of $1.39 \mathrm{~mm}_{\text {period }}$ and $R^{2}=0.98$ for force power and a maximum of $1.39 \mathrm{~mm}_{\text {period }}$ and $R^{2}=0.97$ for roughness. A linear fit to the initial $(<1.0$ $\mathrm{mm}$ texture period) and final $(>1.0 \mathrm{~mm}$ texture period) portions of each curve with slopes as follows: Ascending Power $=1.76$ $\mathrm{N} / \mathrm{mm}_{\text {period }}, R^{2}=0.83$, Ascending Roughness $=1.38, R^{2}=0.80$, Descending Power $=-0.98 \mathrm{~N} / \mathrm{mm}_{\text {period }}, R^{2}=0.97$, Descending Roughness $=-0.78, R^{2}=0.97$.

roughness and each of the physical properties investigated. (This correlation is independent of the scaling of roughness.) This comparison indicates that the total power in the force signal is capable of explaining more of the variance in the roughness psychophysical function than any other property (see Table II). Stepwise multilinear regression revealed that the variance in the psychophysical function for roughness was almost entirely accounted for by the total power in the force signal,with an $\mathrm{R}$ value of $0.984, \mathrm{p}<0.001$.

\begin{tabular}{|c|c|c|c|}
\hline Physics Parameter & $\begin{array}{c}\text { Sum of Squares } \\
\text { Difference }\end{array}$ & $\begin{array}{c}\text { Correlation } \\
\text { Coefficient }\end{array}$ & p-value \\
\hline Mean Position & 0.0415 & 0.12 & $>0.05$ \\
Velocity & 0.0418 & 0.50 & $<0.005$ \\
Kinetic Energy & 0.1287 & 0.03 & $>0.05$ \\
Position Power & 0.0832 & -0.30 & $>0.05$ \\
Acceleration & 0.0297 & 0.78 & $<0.001$ \\
Mean Force & 0.0424 & -0.18 & $>0.05$ \\
Maximum Force PSD & 0.0765 & 0.23 & $>0.05$ \\
Freq. Max. Force & 0.0195 & 0.76 & $<0.001$ \\
Force PSD FA1 Power & 0.0578 & 0.65 & $<0.001$ \\
Force PSD FA2 Power & 0.7514 & 0.45 & $<0.01$ \\
Force PSD Total Power & 0.0046 & 0.98 & $<0.001$ \\
\hline
\end{tabular}

TABLE II

CORRELATION COEFFICIENTS BETWEEN THE PSYCHOPHYSICAL ROUGHNESS FUNCTION AND FUNCTIONS RELATING VARIOUS PHYSICAL PARAMETERS TO SPATIAL PERIOD IN THE STIMULUS (OVER SPATIAL PERIODS FROM 0.025-6.0 MM). SUM OF SQUARES DIFFERENCE IS THE TOTAL OF SQUARED POINT-BY-POINT DIFFERENCES BETWEEN THE FUNCTIONS, DIVIDED BY THE SQUARED VALUES OF THE ROUGHNESS FUNCTION FOR NORMALIZATION. 


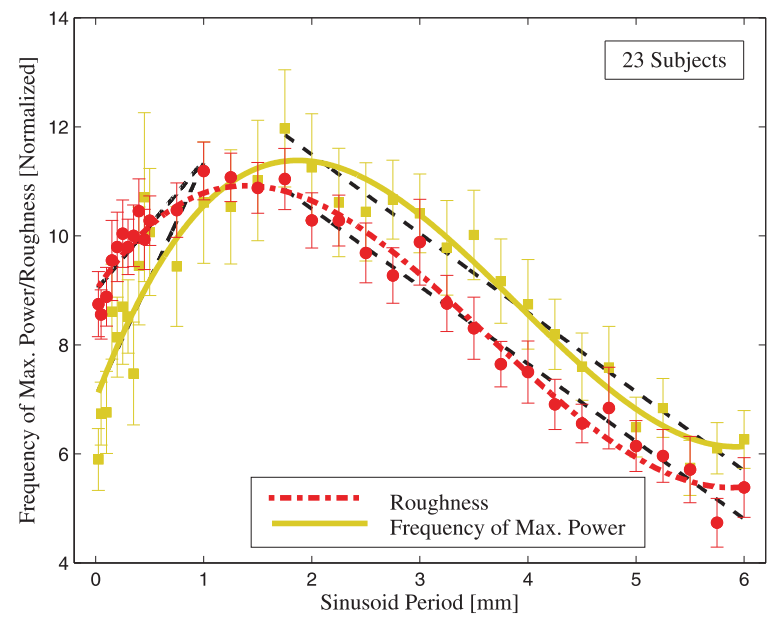

Fig. 11. Roughness estimation function compared with the frequency at which the maximum $z$-axis force signal, as measured by the power spectral density, is found. Frequency and roughness are normalized for comparison. A third order fit to each data set is shown with a maximum at $1.88 \mathrm{~mm}_{\text {period }}$ and $R^{2}=0.89$ for frequency and a maximum at $1.39 \mathrm{~mm}_{\text {period }}$ and $R^{2}=0.97$ for roughness. A linear fit to the initial $(<1.0 \mathrm{~mm}$ texture period $)$ and final ( $>1.75 \mathrm{~mm}$ texture period) portions of each curve with slopes as follows: Initial frequency $=4.28 \mathrm{~Hz} / \mathrm{mm}_{\text {period }}, R^{2}=0.63$, Initial Roughness $=2.38, R^{2}=0.80$, Final frequency $=-1.45$ $\mathrm{N} / \mathrm{mm}_{\text {period }}, R^{2}=0.96$, Final Roughness $=-1.42, R^{2}=0.97$. Outliers greater than $10 \mathrm{x}$ the mean over subjects were removed from the frequency data.

\section{G. Frequency Distribution of Power}

Although these results indicate that the total signal power of force is closely coupled with roughness, it is important to note that the power is not evenly distributed across the frequency spectrum. One-way ANOVAs showed significant effects of sinusoid period on the frequency at which the maximum $z$-axis force signal occurred, or freq $q_{\max },(\mathrm{F}(32,726)=4.22, \mathrm{p}=0.00)$ and on maximum $z$-axis force signal power itself, or force $_{\max }$ $(\mathrm{F}(32,726)=3.15, \mathrm{p}<0.001)$. The form of these dependencies is seen in Fig. 11 and Fig. 12, respectively. The similarity in the shape of the functions, together with the location of the peak of both freq $_{\max }$ and force $_{\max }$ at texture periods higher than that of roughness, suggesting that the two are coupled. That is, a texture surface geometry that produces a higher-frequency power also produces a greater maximum force signal. Variations in roughness lag variations in these coupled signals, across the range of sinusoidal periods.

\section{FREQUENCy Distribution of Power AND MECHANORECEPTOR POPULATIONS}

Given that the frequencies that primarily contribute to power can be identified from the data, we can ask whether the observed frequencies are related to the sensitivity of particular mechanoreceptor populations, particularly those that are sensitive to vibration. In particular, the force signal can be partitioned into two bands of frequency: $5-50 \mathrm{~Hz}$, a response range roughly that of the FA1 receptors, and $>50 \mathrm{~Hz}$, a range associated with the FA2 receptors. For ease of exposition, these will be labeled the FA1 and FA2 bands, respectively. The

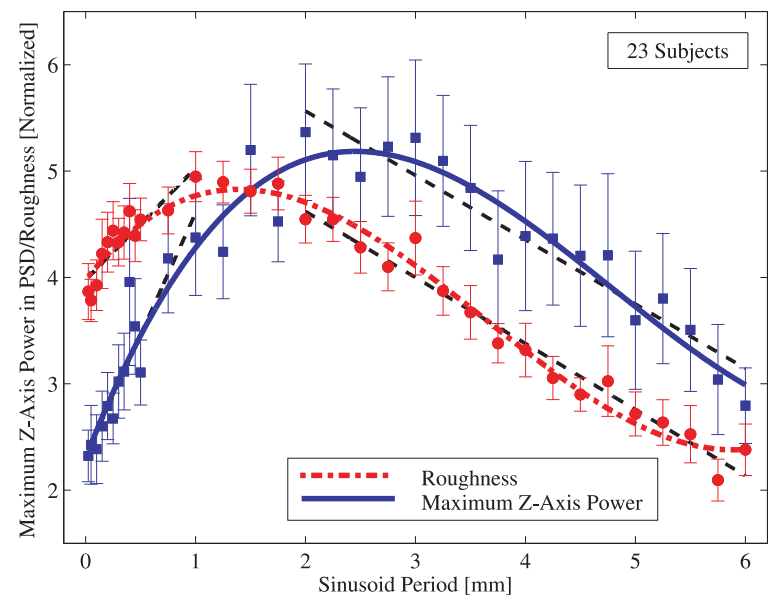

Fig. 12. Roughness function compared with the maximum $z$-axis force signal, as measured by the power spectral density. Maximum force and roughness are normalized for comparison. A third order fit to each data set is shown with a maximum at $2.45 \mathrm{~mm}_{\text {period }}$ and $R^{2}=0.93$ for frequency and a maximum at $1.39 \mathrm{~mm}_{\text {period }}$ and $R^{2}=0.97$ for roughness. A linear fit to the initial $(<1.0 \mathrm{~mm}$ texture period) and final $(>2.00 \mathrm{~mm}$ texture period) portions of each curve with slopes as follows: Initial $\mathrm{PSD}=2.29 \mathrm{~N} / \mathrm{mm}_{\text {period }}$, $\mathrm{R}^{2}=0.86$, Initial Roughness $=1.05, \mathrm{R}^{2}=0.80$, Final $\mathrm{PSD}=-0.61$ $\mathrm{N} / \mathrm{mm}_{\text {period }}, \mathrm{R}^{2}=0.91$, Final Roughness $=-0.62, \mathrm{R}^{2}=0.97$. Outliers greater than $10 \mathrm{x}$ the mean over subjects were removed from the PSD data.

power in the force signal can then determined for each of these bands for each experimental trial. For each sinusoidal texture period this band-limited force signal power was averaged over subjects and iterations. One-way ANOVAs showed an effect of texture period on $z$-axis force signal power in both ranges (FA1: $\mathrm{F}(32,726)=7.79, \mathrm{p}<0.001, \mathrm{FA} 2: \mathrm{F}(32,726)=77.62, \mathrm{p}<$ $.001)$.

The band-limited force signal power function for the socalled FA1 and FA2 ranges can be seen in Figs 13 and 14. The psychophysical roughness function is shown for comparison, again normalized to the force signal. It is clear that neither the FA1 nor FA2 bandwidths coincides with the roughness function as well as the PSD total power (see Table II) although the FA1 function demonstrates a similarly shaped curve with a peak that lags that of roughness.

To further pursue the relationship between force frequency power and roughness, we asked what portion of the periodogram best accounts for roughness judgments. The power in a $20-\mathrm{Hz}$ window spanning a base frequency $f$ to $f+$ $20 \mathrm{~Hz}$, was correlated with roughness across all trials, to produce a correlation for the given $f$. This was repeated by moving $f$ across the frequency range from low to high. As shown in Fig. 15, as the window moves across the frequency range of $500 \mathrm{~Hz}$ from low to high, the peak in correlation (approximately 0.90) occurs when the window is at 20-40 $\mathrm{Hz}$, which is well within the bandwidth associated with FA1 receptors but below that of FA2s.

A further analysis asked whether the power within the FA1 band is sufficient to account for roughness judgments. That is, does the higher-frequency power contribute at all, or might it even introduce noise and reduce the correlation between 


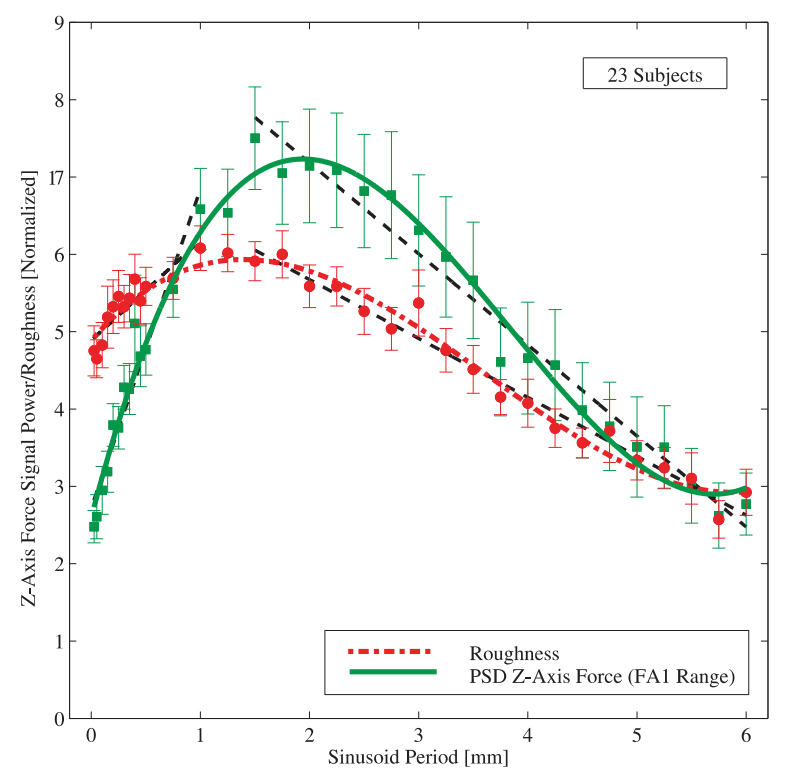

Fig. 13. Power Spectral Density of $z$-axis forces within the approximate FA1 receptor frequency range $(5-50 \mathrm{~Hz})$, compared to roughness as a function of sinusoid period. PSD and roughness are normalized for comparison. A third order fit to each data set is shown with a maximum of $1.94 \mathrm{~mm}_{\text {period }}$ and $\mathrm{R}^{2}=0.97$ for PSD and a maximum of $1.39 \mathrm{~mm}_{\text {period }}$ and $\mathrm{R}^{2}=0.97$ for roughness. A linear fit to the initial $(<1.0 \mathrm{~mm}$ texture period) and final $(>1.5 \mathrm{~mm}$ texture period) portions of each curve with slopes as follows: Initial $\mathrm{PSD}=4.12$ $\mathrm{N} / \mathrm{mm}_{\text {period }}, \mathrm{R}^{2}=0.93$, Initial Roughness $=1.29, \mathrm{R}^{2}=0.80$, Final $\mathrm{PSD}=-1.17 \mathrm{~N} / \mathrm{mm}_{\text {period }}, \mathrm{R}^{2}=0.97$, Final Roughness $=-0.76$, $\mathrm{R}^{2}=0.97$.

power and roughness? Cholewiak et al. [34] have shown that higher-frequency components can enhance threshold detection. To address this question, we held $f$ constant at $5 \mathrm{~Hz}$ to anchor the lower end of a frequency window in which power was accumulated. As the window's higher end moved across the frequency range in $1-\mathrm{Hz}$ increments to a maximum of $500 \mathrm{~Hz}$, expanding the window in which power was accumulated, we examined the correlation across trials between roughness and total power.

Figure 16 shows the results of this analysis. Correlations were low and variable until the window's upper end reached approximately $15 \mathrm{~Hz}$. Beyond that, the correlation coefficient rose steadily to reach to $>0.80$ at approximately $100 \mathrm{~Hz}$, $\mathrm{p}<0.01$. Most of the signal that for roughness perception appears to be coming from frequencies below $50 \mathrm{~Hz}$ (FA1 band), but higher frequencies, up to $350 \mathrm{~Hz}$, appear to be required to achieve correlations of $>0.98$. This result points to some value within the FA2 range.

One possibility is that the contribution of high- and lowfrequency FA receptors varies with the density of the stimulus elements. Similar specialization of receptors according to surface properties is proposed by the duplex mode of texture perception via the bare finger [18] (although in that case slowly adapting receptor populations are implicated). If FA receptors partition the textural range for sinusoidal surfaces explored with a probe, a natural expectation is that textures with small periods generate more high-frequency vibration and lie within the FA2 bandwidth, whereas textures with large periods

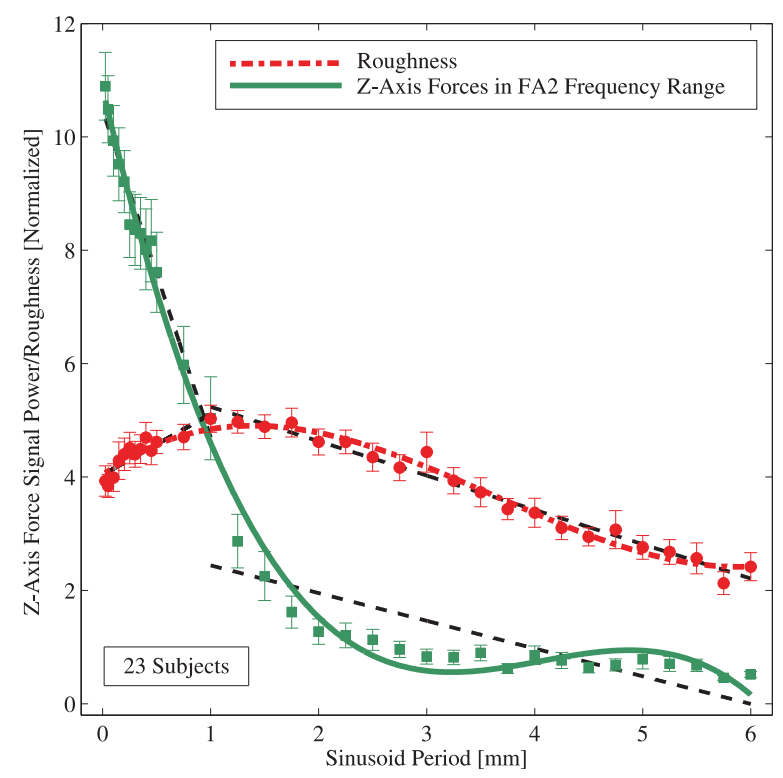

Fig. 14. Power Spectral Density of $z$-axis forces within the FA2 receptor frequency range $(50-500 \mathrm{~Hz})$, compared to roughness as a function of sinusoid period. PSD and roughness are normalized for comparison. A third order fit to each data set is shown with a maximum at $0.025 \mathrm{~mm}_{\text {period }}$ and $R^{2}=0.99$ for PSD and a maximum at $1.39 \mathrm{~mm}_{\text {period }}$ and $R^{2}=0.97$ for roughness. A linear fit to the initial $(<1.0 \mathrm{~mm}$ texture period) and final $(>1.0 \mathrm{~mm}$ texture period) portions of each curve with slopes as follows: Initial $\mathrm{PSD}=-5.77 \mathrm{~N} / \mathrm{mm}_{\text {period }}, \mathrm{R}^{2}=0.96$, Initial Roughness $=0.80$, $\mathrm{R}^{2}=0.80$, Final PSD $=-0.49 \mathrm{~N} / \mathrm{mm}_{\text {period }}, \mathrm{R}^{2}=0.52$, Final Roughness $=-0.61, \mathrm{R}^{2}=0.97$.

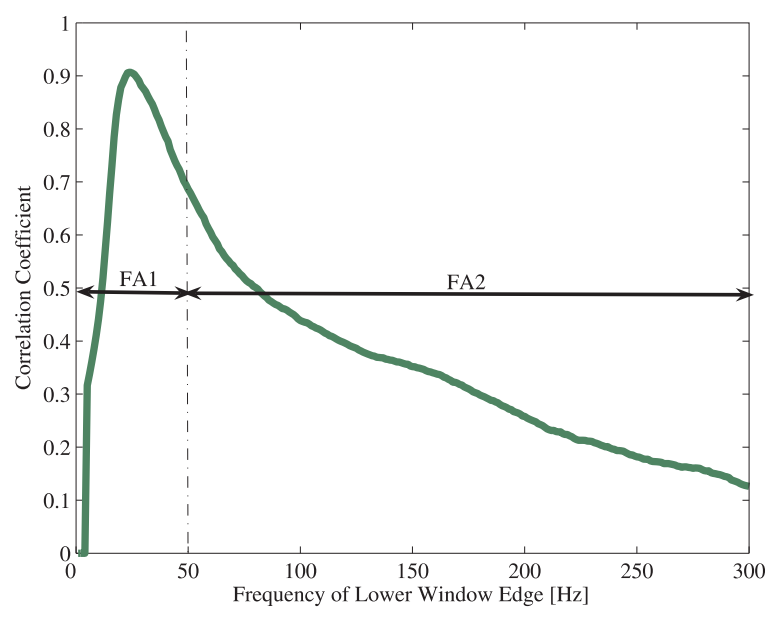

Fig. 15. Correlation coefficient between subjective roughness and the power in the force signal in a $20 \mathrm{~Hz}$ window sliding across the PSD periodogram. The window's lower edge is at the frequency displayed on the $x$ axis. The FA1 and FA2 frequency bandwidths are indicated with arrows.

generate more low-frequency vibrations and excite the FA1 receptors. Accordingly, roughness for small periods should be more influenced by the power in the higher frequency band of the force signal, and roughness for larger periods by the power in the lower frequency range.

To test this hypothesis, the relation between roughness and power of the force signal was again examined with windows of increasing size, but separately for textures with relatively 


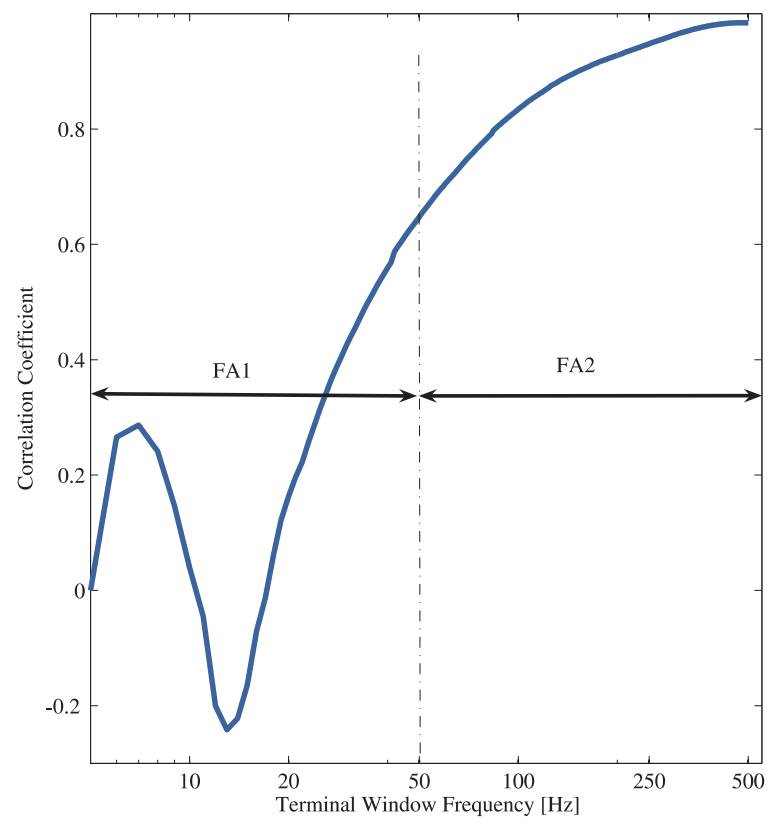

Fig. 16. Correlation coefficient between subjective roughness and the power in the force signal in a frequency window of increasing size. The window's lower frequency is fixed at $5 \mathrm{~Hz}$. The correlation coefficient is plotted against the the window's upper frequency which is used as the independent variable. The FA1 and FA2 frequency bandwidths are indicated with arrows.

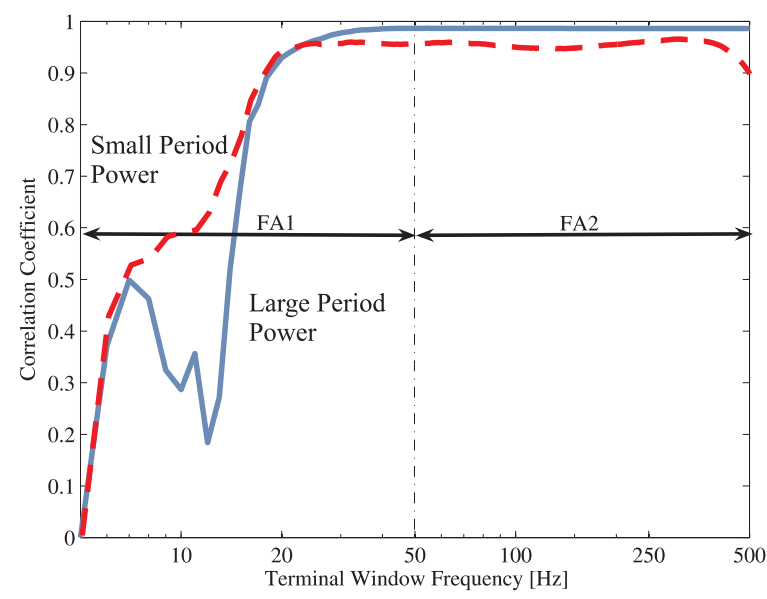

Fig. 17. Correlation coefficient between subjective roughness and the power in the force signal for small period $(0.025-0.5 \mathrm{~mm})$ and large period $(2.0-6.0 \mathrm{~mm})$ sinusoidal textures in a frequency window of increasing size. The window's lower frequency is fixed at $5 \mathrm{~Hz}$. The correlation coefficient is plotted against the window's upper frequency, which is used as the independent variable.

small and large periods. For these purposes, texture ranges were selected where the roughness function was approximately linear with spatial period: $0.025-0.5 \mathrm{~mm}$ for the small range, and $2.0-6.0 \mathrm{~mm}$ for the largest. Fig. 17 shows the results for textures with small and large periods. The data give little support to the idea that high-frequency information contributes more to the textures with small spatial periods. Contrary to expectations, power within frequency windows of less than $50 \mathrm{~Hz}$ was highly correlated with roughness for both large and small period textures. Moreover, the textures with small periods actually evidenced a stronger correlation with lowfrequency power than those with large periods, and the peak correlation was reached somewhat lower in the frequency range for the finer textures than the coarse. Thus it appears that the predominance of low-frequency information in determining roughness holds across the geometric variations in the stimuli.

\section{DISCUSSION}

The present research sought an account of the perceived roughness of sinusoidal surfaces explored with a probe, in terms of the physical variables concomitant with exploration. The variables that were examined included kinematics (probe position, velocity and acceleration) and dynamic physical properties (force variability, mean force, kinetic energy and power in the force signal). The initial analysis focused on how these parameters change with sinusoidal period and correlated the variations with estimates of perceived roughness. Ultimately, the power in the z-axis force signal was found to be strongly related to the roughness judgment across a broad range of geometric variation. Further detailed investigation of the vibratory signal implicated the low-frequency component, theoretically associated with the FA1 mechanoreceptors, as most critical across the stimulus range.

Convergent evidence for this conclusion was found in experiments that used texture elements in the shape of truncated cones, both regularly spaced and in randomly dithered arrangements, described in [14]. The probe was rendered as having a spherical tip with four radius values between 0.25 and $1.5 \mathrm{~mm}$. As the number of rendered stimuli in those studies was much smaller than in the experiment reported here (11 vs. 33 in the experiment with SGTs), the correlations are less reliable, and inferences are limited. Indeed, in both studies with conical elements, there were stronger correlations between all the physical parameters and roughness than in the current study with sinusoids, but correlations between the roughness function and the $z$-axis total power function were again high: .94 or greater in both conical-texture studies for all probe sizes.

The present results confirm earlier observations in the literature that point to force variability as critical to roughness perception through a probe [26], [27], [28]. While Yoshioka et. al converged on power as the underlying variable, Otaduy and Lin chose acceleration. The latter is not surprising, as the total power can be seen as a measure of the variability of the force to which a subject's fingers are exposed as they move the manipulandum across a textured surface. One would expect, then, that instantaneous acceleration would correlate moderately well with roughness, since it, too, provides a measure of the variability of force. It is also understandable that the correlation is much better for the textures with larger periods, since instantaneous acceleration is determined from the second derivative of position and is subject to noise, particularly in the high frequency range of the spectrum.

\section{CONCLUSION}

This research supports a physical account of the roughness judgment when people explore sinusoidal surfaces with a 
probe, in terms of the power in the z-axis force signal. Moreover, the low-frequency component of the vibratory signal appears to carry greatest weight, regardless of the geometry of the stimuli. In addition to contributing to our understanding of the perceived roughness of textures explored through a rigid probe, the present study points to the value of highfidelity haptics for rendering such surfaces. Even the finest textures rendered here, which pushed at the boundaries of device limitations, appear to have produced the impression of an underlying surface with tangible roughness.

\section{ACKNOWLEDGMENT}

The authors would like to thank the National Science Foundation for its support under Grants IRI-9420869 and IIS9802191. Roberta Klatzky also thanks the Alexander Von Humboldt Society for facilitating her residence in Munich, Germany during the preparation of this manuscript.

\section{REFERENCES}

[1] C.J. Cascio and K. Sathian, "Temporal Cues Contribute to Tactile Perception of Roughness," J. Neuroscience, vol. 21, no. 14, pp. 52895296, July 2001.

[2] C.E. Connor and K.O. Johnson, "Neural Coding of Tactile Texture: Comparison of Spatial and Temporal Mechanisms for Roughness Perception," J. Neuroscience, vol. 12, no. 9, pp. 3414-3426, Sept. 1992.

[3] R.L. Klatzky and S.J. Lederman, "Tactile Roughness Perception with a Rigid Link Interposed between Skin and Surface," Perception and Psychophysics, vol. 61, no. 4, pp. 591-607, 1999.

[4] M. Lawrence, R. Kitada, R. Klatzky, and S. Lederman, "Haptic Roughness Perception of Linear Gratings via Bare Finger or Rigid Probe," Perception, vol. 36, pp. 547-557, 2007.

[5] S.J. Lederman, "Tactile Roughness of Grooved Surfaces: The Touching Process and Effects of Macro- and Microsurface Structure," Perception and Psychophysics, vol. 16, no. 2, pp. 385- 395, 1974.

[6] E.M. Meftah, L. Belingard, and C.E. Chapman, "Relative Effects of the Spatial and Temporal Characteristics of Scanned Surfaces on Human Perception of Tactile Roughness Using Passive Touch," Experimental Brain Research, vol. 132, pp. 351-361, 2000.

[7] A.M. Smith, C.E. Chapman, M. Deslandes, J.-S. Langlais, and M.-P. Thibodeau, "Role of Friction and Tangential Force Variation in the Subjective Scaling of Tactile Roughness," Experimental Brain Research, vol. 144, pp. 211-223, 2002.

[8] M. Taylor and S. Lederman, "Tactile Roughness of Grooved Surfaces: A Model and the Effect of Friction," Perception and Psychophysics, vol. 17, no. 1, pp. 23-36, 1975.

[9] D. Kornbrot, P. Penn, H. Petrie, S. Furner, and A. Hardwick, "Roughness Perception in Haptic Virtual Reality for Sighted and Blind People," Perception and Psychophysics, vol. 69, no. 4, pp. 502- 512, 2007.

[10] M.R. McGee, P. Gray, and S. Brewster, "Haptic Perception of Virtual Roughness," Proc. ACM Conf. Human Factors in Computing Systems (CHI 01), pp. 155-156, Mar./Apr. 2001.

[11] M. Minsky and S. J. Lederman. "Simulated haptic textures: Roughness, Proc. ASME Dynamic Systems and Control Division, vol. 58, pp. 421426, 1996.

[12] P. Penn, D. Kornbrot, S. Furner, A. Hardwick, C. Colwell, and H. Petrie, "Roughness Perception in Haptic Virtual Reality: The Impact of the Haptic Device, Endpoint and Visual Status," Unpublished manuscript, 2004.

[13] B. Unger, R. Hollis, and R. Klatzky, "The Geometric Model for Perceived Roughness Applies to Virtual Textures," Proc. IEEE Symp. Haptic Interfaces for Virtual Environment and Teleoperator Systems, pp. 3-10, Mar. 2008.

[14] B. Unger, R., Hollis, and R. Klatzky. "Roughness perception in virtual textures," IEEE Transactions on Haptics, vol. 4, no. 2, pp.122-133, 2011.

[15] R. L. Klatzky and S. J. Lederman, "Multisensory texture perception". In J. Kaiser and M. Naumer (Eds.), Multisensory Object Perception in the Primate Brain, pp. 211-230. New York: Springer, .2010.

[16] M. Hollins, F. Lorenz, and D. Harper, "Somatosensory Coding of Roughness: The Effect of Texture Adaptation in Direct and Indirect Touch,” J. Neuroscience, vol. 26, no. 20, pp. 5582-5588, May 2006.
[17] M. Hollins, S.J. Bensmaa, and S. Washburn, "Vibrotactile Adaptation Impairs Discrimination of Fine, but Not Coarse, Textures," Somatosensory and Motor Research, vol. 18, no. 4, pp. 253-262, 2001.

[18] M. Hollins and S.R. Risner, "Evidence for the Duplex Theory of Tactile Texture Perception," Perception and Psychophysics, vol. 62, no. 4, pp. 695-705, 2000.

[19] S. J. Bolanowski, G. A. Gescheider, R. T. Verillo, and C. M. Checkosky. "Four channels mediate the mechanical aspects of touch," J. Acoustic Society of America, 84(5):16801694, 1988.

[20] K. Johnson. "Neural basis of haptic perception," Stevens Handbook of Experimental Psychology, chapter 13, pages 537 583. Wiley, 2002.

[21] A. B. Valbo and R. S. Johansson. "Properties of cutaneous mechanoreceptors in the human hand related to touch sensation" Human Neurobiology, vol. 3, pp. 314, 1984.

[22] R. Klatzky, S. Lederman, C. Hamilton, and G. Ramsay, "Perceiving Roughness via a Rigid Probe: Effects of Exploration Speed," Proc. ASME Dynamic Systems and Control Division 99, pp. 27-33, 1999.

[23] R.L. Klatzky, S.J. Lederman, C. Hamilton, M. Grindley, and R.H. Swendsen, "Feeling Textures through a Probe: Effects of Probe and Surface Geometry and Exploratory Factors," Perception and Psychophysics, vol. 65, no. 4, pp. 613-631, 2003.

[24] S. Lederman and R. Klatzky, "Sensing and Displaying Spatially Distributed Fingertip Force in Haptic Interfaces for Teleoperator and Virtual Environment Systems," Presence, vol. 8, no. 1, pp. 86- 103, Feb. 1999.

[25] S.A. Wall and W.S. Harwin, "Effect of Physical Bandwidth on Perception of Virtual Gratings," Proc. ASME Dynamic Systems and Control Division (Symp. Haptic Interfaces for Virtual Environments and Teleoperators), pp. 1033-1039, 2000.

[26] M. A. Otaduy, N. Jain, A. Sud, and M. C. Lin. "Haptic display of interaction between textured models," Proc. IEEE Visualization, pp. 297 304, 2004.

[27] M. A. Otaduy and M. C. Lin. "A perceptually-inspired force model for haptic texture rendering, Proc. 1st Symposium on applied perception in graphics and visualization, pages 123126. ACM Press, 2004.

[28] T. Yoshioka, S.J. Bensmaia, J.C. Craig, and S.S. Hsiao. "Texture perception through direct and indirect touch: An analysis of perceptual space for tactile textures in two modes of exploration," Somatosensory and Motor Research, 24(1-2):5370, March-June 2007.

[29] S. Bensmaa and M. Hollins. "Pacinian representation of fine surface texture," Perception and Psychophysics, vol. 67, no.5, pp. 842-854, 2005.

[30] S. Bensmaia, M. Hollins, and J. Yau. "Vibrotactile intensity and frequency information in the Pacinian system: A psychophysical model," Perception and Psychophysics, vol 67, no. 5, pp. 828-841, 2005.

[31] R. Hollis, S. Salcudean, and A.P. Allan, "A Six-Degree-of-Freedom Magnetically Levitated Variable Compliance Fine Motion Wrist: Design, Modeling, and Control," IEEE Trans. Robotics and Automation, vol. 7, no. 3, pp. 319-332, June 1991.

[32] P. Berkelman and R. Hollis, "Lorentz Magnetic Levitation for Haptic Interaction: Device Design, Performance, and Integration with Physical Simulations," Intl J. Robotics Research, vol. 19, no. 7, pp. 644-667, July 2000 .

[33] P.J. Berkelman, "Tool-Based Haptic Interaction with Dynamic Physical Simulations Using Lorentz Magnetic Levitation," PhD dissertation, Carnegie Mellon Univ., The Robotics Inst., 1999.

[34] S. A. Choleiak, K. Kwangtaek, H. Z. Tan, and B. D. Adelstein. "A Frequency-Domain Analysis of Haptic Gratings," IEEE TRans. Haptics, vol. 3, no. 1, pp. 3-14, 2010.

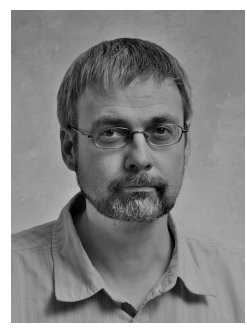

Bertram Unger (MD University of Manitoba, $\mathrm{PhD}$ Carnegie Mellon University, Robotics) is currently Assistant Professor and Research Director of the Clinical Learning and Simulation Facility in the Faculty of Medicine at the University of Manitoba . $\mathrm{He}$ is also a clinician with the University's Department of Internal Medicine, Section of Critical Care and has an adjunct appointment with its Faculty of Engineering. He holds his PhD. from the Robotics Institute at Carnegie Mellon University and recently completed a post-doctoral Fellowship at the University of Pittsburgh in the Faculty of Bioengineering. 


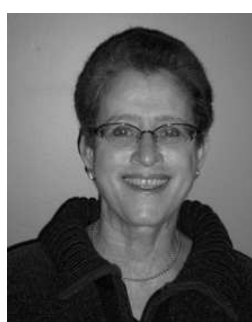

environments.

Roberta Klatzky ( $\mathrm{PhD}$ Stanford, Psychology) is Professor of Psychology and Human Computer Interaction at Carnegie Mellon University. Her research interests are in human perception and cognition, with special emphasis on spatial cognition and haptic perception. She has done extensive research on human haptic and visual object recognition, navigation under visual and nonvisual guidance, and perceptually guided action, with application to navigation aids for the blind, haptic interfaces, exploratory robotics, image-guided surgery, and virtual

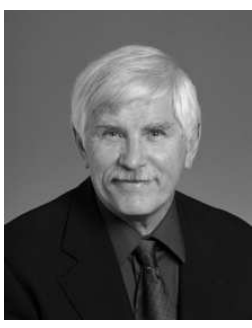

Ralph Hollis (PhD University of Colorado, Physics) is Research Professor of Robotics and Electrical and Computer Engineering at Carnegie Mellon University. Dr. Hollis was a Research Staff Member at the Thomas J. Watson Research Center from 197893 where he worked in magnetism, acoustics, and robotics, and was Manager of Advanced Robotics from 1986-93. He is a member of the American Physical Society and a Fellow of IEEE. He is founding director of the Microdynamic Systems Laboratory at Carnegie Mellon University where his research centers on haptics, agile manufacturing, and dynamically-stable mobile robots. 